\title{
Charge-cluster glass in an organic conductor
}

\author{
F. Kagawa ${ }^{1,2 \star}$, T. Sato ${ }^{1}$, K. Miyagawa ${ }^{1}$, K. Kanoda ${ }^{1}$, Y. Tokura ${ }^{1,3}$, K. Kobayashi ${ }^{4}$, R. Kumai ${ }^{2,4}$ \\ and Y. Murakami ${ }^{4}$
}

\begin{abstract}
Geometrically frustrated spin systems often do not exhibit long-range magnetic ordering, resulting in either quantummechanically disordered states, such as quantum spin liquids ${ }^{1}$, or classically disordered states, such as spin ices ${ }^{2,3}$ or spin glasses 4 . Geometric frustration may play a similar role in charge ordering 5,6 , potentially leading to unconventional electronic states without long-range order; however, there are no previous experimental demonstrations of this phenomenon. Here, we show that a charge-cluster glass evolves on cooling in the absence of long-range charge ordering for an organic conductor with a triangular lattice. A combination of timeresolved transport measurements and $X$-ray diffraction reveals that the charge-liquid phase has two-dimensional charge clusters that fluctuate extremely slowly $(<10-100 \mathrm{~Hz})$ and heterogeneously. On further cooling, the cluster dynamics freezes, and a charge-cluster glass is formed. Surprisingly, these observations correspond to recent ideas regarding the structural glass formation of supercooled liquids ${ }^{7-10}$. Glassy behaviour has often been found in transition-metal oxides, but only under the influence of randomly located dopants ${ }^{11,12}$. As organic conductors are very clean systems, the present glassy behaviour is probably conceptually different.
\end{abstract}

Wigner-type charge ordering is a phenomenon in which an equal number of charge-rich and charge-poor sites occupy a lattice such that rich-rich (or poor-poor) neighbouring pairs are avoided as much as possible. However, in a geometrically frustrated lattice, this constraint is insufficient to determine a specific charge ordering among the various charge configurations, analogously to spin-frustrated systems (Fig. 1a); thus, the geometric frustration potentially undermines the tendency towards long-range charge ordering, as was first suggested in ref. 5. As a result, exotic electronic states may be exhibited at low temperatures when long-range charge ordering is avoided.

The material investigated in this study is the organic conductor $\theta$-(BEDT-TTF $)_{2} \mathrm{RbZn}(\mathrm{SCN})_{4}$ (denoted $\theta$-RbZn), where BEDTTTF (ET) denotes bis(ethylenedithio)tetrathiafulvalene ${ }^{13}$. The crystal structure consists of alternating layers of conducting ET molecules and insulating anions, and the ET molecules form a geometrically frustrated triangular lattice (Fig. 1b), where the ratio of two different inter-site Coulomb interactions is calculated to be $\sim 0.86$ (ref. 14). The ET conduction band is hole-1/4filled; thus, the charge-delocalized state (the charge-liquid phase) is subject to charge ordering instability ${ }^{15}$. However, the charge frustration created by the triangular lattice may prevent longrange ordering. Experimentally, a structural transition with the modulation $\mathbf{q}_{\mathrm{o}}=\left(\begin{array}{lll}0 & 0 & 1 / 2\end{array}\right)$ occurs at $\sim 200 \mathrm{~K}$ and relaxes the degree of charge frustration; consequently, a horizontal charge order that matches $\mathbf{q}_{\mathrm{o}}$ is stabilized ${ }^{16-18}$. This charge order is a strong first-order
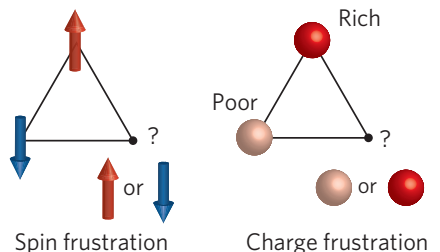

b

Charge frustration
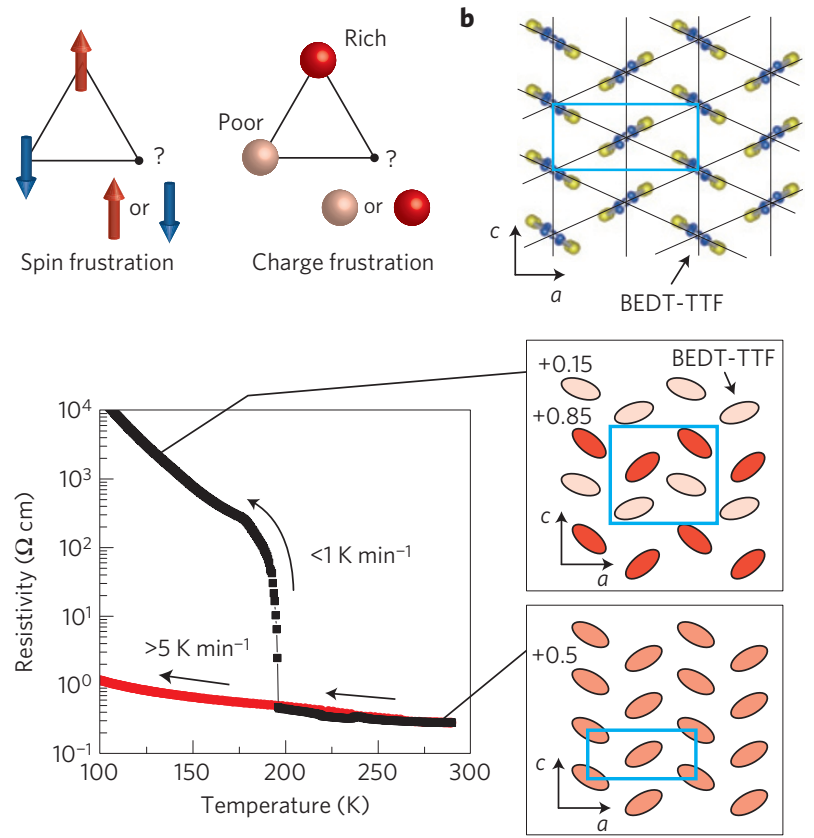

Figure 1 | Charge frustration and crystal structure of

$\boldsymbol{\theta}$-(BEDT-TTF) $\left.{ }_{2} \mathbf{R b Z n ( S C N}\right)_{4} \cdot \mathbf{a}$, An illustration of the analogy between spin frustration and charge frustration. $\mathbf{b}$, The structure of the BEDT-TTF layer. The lattice parameters are $a \approx 10.2 \AA$ and $c \approx 4.6 \AA$ at $220 \mathrm{~K}$ (ref. 16).

c, The temperature dependence of the resistivity during cooling for different temperature-sweeping rates. The insets indicate the crystal structures of the high-temperature phase (lower inset) and the low-temperature phase (upper inset). Colours as for a. In the upper inset, the charge-ordering pattern is also shown. To emphasize the two-fold structural modulation of the $c$ axis, the upper inset is depicted in an exaggerated manner. In each panel, the unit cell is indicated by a blue rectangle.

transition accompanied by a sudden increase in resistivity (Fig. 1c). When rapidly cooled $\left(>5 \mathrm{~K} \mathrm{~min}^{-1}\right)$, the charge/structure order at $200 \mathrm{~K}$ vanishes, giving way to a charge-liquid phase with a frustrated triangular lattice, which is maintained at lower temperatures ${ }^{19,20}$ (Fig. 1c). As there is no resistivity anomaly at $200 \mathrm{~K}$ during rapid cooling (Fig. 1c), the phase exhibited above $200 \mathrm{~K}$ is considered a continuation of the rapidly cooled phase. The electronic states above $200 \mathrm{~K}$ may be exotic, as suggested in the literature: the optical conductivity measurements revealed that the charge liquid cannot be ascribed to a conventional metal that has a Drude response ${ }^{21}$. More interestingly, previous NMR measurements, which are a probe of local spin dynamics, implied the existence of slow charge

\footnotetext{
${ }^{1}$ Department of Applied Physics, University of Tokyo, Tokyo 113-8656, Japan, ${ }^{2}$ CREST, Japan Science and Technology Agency (JST), Tokyo 102-0076, Japan, ${ }^{3}$ RIKEN Center for Emergent Matter Science (CEMS), Wako 351-0198, Japan, ${ }^{4}$ Condensed Matter Research Center (CMRC) and Photon Factory, Institute of Materials Structure Science, High Energy Accelerator Research Organization (KEK), Tsukuba 305-0801, Japan. *e-mail: kagawa@ap.t.u-tokyo.ac.jp
} 
a

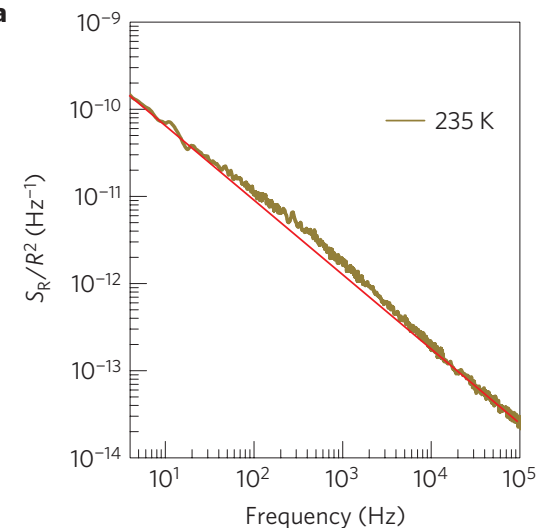

d

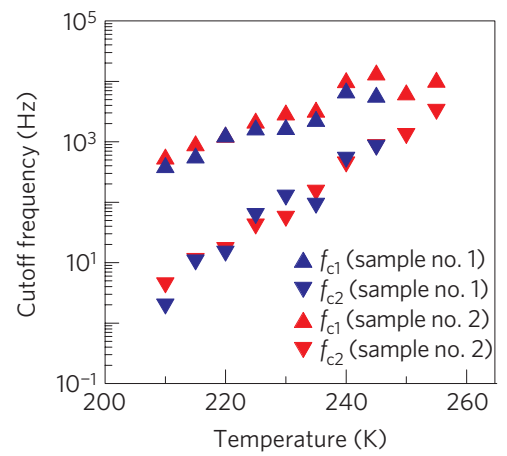

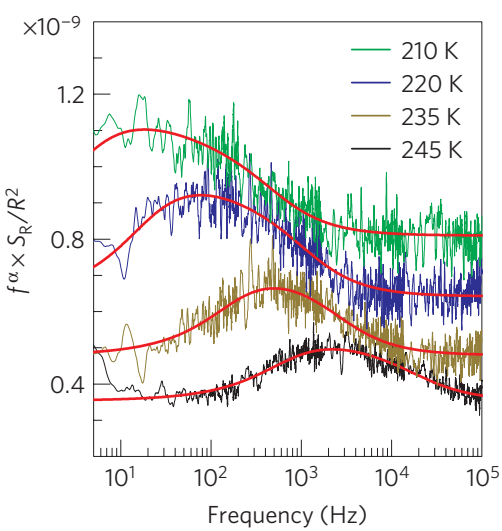

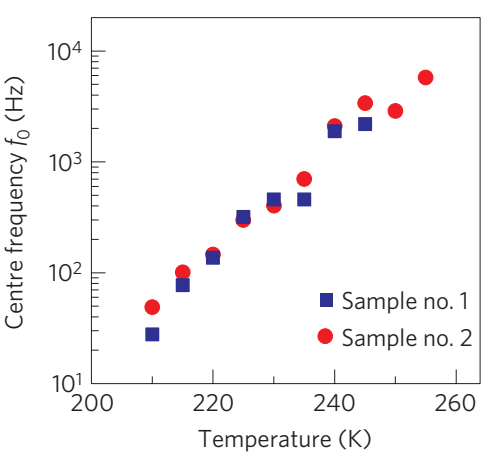

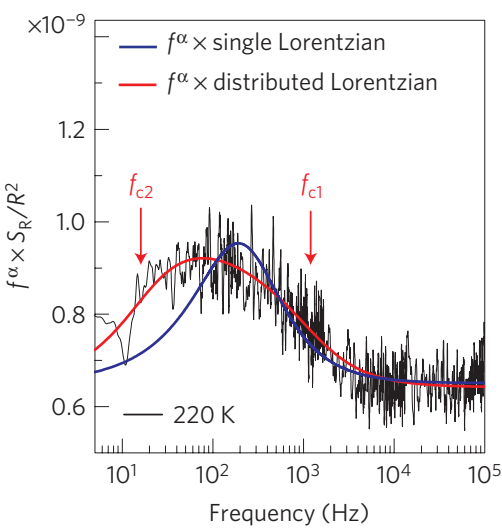

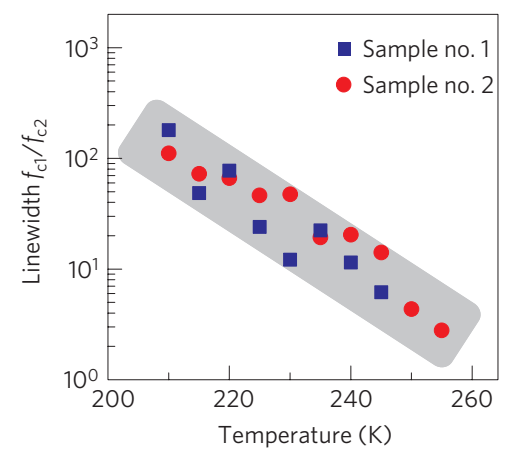

Figure 2 | Resistance fluctuations in the charge-liquid phase. a, A typical resistance power spectrum density $S_{R}$ normalized by the resistance squared $R^{2}$. $S_{R} / R^{2}$ is found to be independent of an applied current as long as the current is low enough, indicating that the detected fluctuations reflect the nature of the equilibrium state (see also Supplementary Fig. S3). b, Power spectra densities for various temperatures with $f^{\alpha} \times S_{R} / R^{2}$ representations. The red lines are fits to the distributed Lorentzian model (Supplementary Information). c, A comparison of the $S_{R} / R^{2}$ characterizations using different schemes. The notations $f_{c 1}$ and $f_{c 2}$ represent the high- and low-frequency cutoffs, respectively, in the distributed Lorentzian model. $\mathbf{d}$, Temperature profiles of the fitting parameters $f_{c 1}$ and $f_{c 2}$.e, $\mathbf{f}$, Slowing of the centre frequency $(\mathbf{e})$ and concomitant growth of the dynamic heterogeneity (f). The shaded area in $\mathbf{f}$ is intended as a guide for the eyes.

dynamics of the order of kilohertz above $200 \mathrm{~K}$ (ref. 22). These slow dynamics may indicate that the charge liquid above $200 \mathrm{~K}$, where the triangular lattice is held, is transforming into a classically disordered state, that is, an electronic glass, but the relevance of this result to known glass formers is far from clear at present. To demonstrate possible electronic-glass-forming behaviour, several key concepts need to be tested, for example, the temperature evolution of the slow charge dynamics, the spatial and dynamic heterogeneity, and the electronic-glass transition.

First, to directly detect the evolution of possible slow charge dynamics by determining their frequency, we implemented noise measurements, that is, resistance fluctuation spectroscopy ${ }^{23}$. The typical resistance power spectrum density $S_{\mathrm{R}}$ is shown in Fig. 2a. The global $S_{\mathrm{R}}$ can be well fitted by $f^{-\alpha}$ with $\alpha \sim 0.8-0.9$ (weakly temperature dependent, $f$ denotes frequency). This fitting reflects the prevalent $1 / f$ noise, where the frequency exponent is not necessarily 1 (ref. 24). The origin of the ubiquitous $1 / f$ noise was not identified for the present case and is beyond the scope of this study. Instead, we note that there is a finite deviation from the background $1 / f$ noise over a certain frequency range (Fig. 2a). This deviation indicates that resistance fluctuations with a characteristic frequency are superimposed on the featureless $1 / f$ noise (see Supplementary Information). For clarity, $f^{\alpha} \times S_{\mathrm{R}}$ versus $f$ is plotted for select temperatures in Fig. 2b, where the additional contribution appears as a broad peak rising out of a constant background (for this material, our methods are applicable only above $200 \mathrm{~K}$; see Methods).

On quantifying the non-1/f contributions, we found that the linewidth of the power spectrum density in the $f^{\alpha} \times S_{R}$ representation was broader than the expected linewidth for a single Lorentzian multiplied by $f^{\alpha}$ (Fig. 2c). To evaluate the linewidth, we introduced a hypothetical superposition of continuously distributed Lorentzians with high-frequency $f_{c 1}$ and low-frequency $f_{c 2}$ cutoffs (see Supplementary Information). We found that the spectrum shape is well reproduced using this scheme (Fig. 2b,c). The extracted values for $f_{c 1}$ and $f_{c 2}$ (Fig. 2d) reflect the fastest and slowest fluctuators, respectively, that are relevant to the non- $1 / f$ contributions, from which we can estimate the centre frequency $f_{0}$ (defined as the geometric mean $\left.\left(f_{c 1} f_{c 2}\right)^{1 / 2}\right)$ and the linewidth (defined as the ratio $\left.f_{c 1} / f_{c 2}\right)$. The temperature profiles of $f_{0}$ and $f_{c 1} / f_{c 2}$ are shown in Fig. 2e,f, respectively, and two features can be emphasized. First, $f_{0}$ slows down by several orders of magnitude as the temperature decreases; remarkably, just above the frustration-relaxing structural transition (approximately $200 \mathrm{~K}$ ), the lifetime of the long-lived fluctuator is less than $10 \mathrm{~Hz}$ (Fig. $2 \mathrm{~d}$ ). Second, the ratio $f_{c 1} / f_{c 2}$ noticeably increases, that is, the dynamics become more heterogeneous, as the temperature decreases. These results are reproducible and are therefore considered to arise from the intrinsic nature of $\theta$-RbZn. We note that slow dynamics accompanied by dynamic heterogeneities are known to be key experimental properties for the vitrification process in supercooled normal liquids ${ }^{25}$.

Some simulation results for supercooled normal liquids argued that medium-range crystalline clusters are critical for understanding the heterogeneous slow dynamics at a microscopic level ${ }^{7-10}$, although the importance of these crystalline clusters is controversial $^{26-29}$, and there are still no clear experimental observations of crystalline clusters. To obtain further insight into the observed heterogeneous slow dynamics for the charge liquid, it is 
necessary to determine whether a type of charge cluster evolves with the slow dynamics. We conducted X-ray diffuse scattering measurements. A typical photographic oscillation image is shown in Fig. 3a. As previously reported ${ }^{16}$, diffuse scattering characterized by $\mathbf{q}_{\mathrm{d}} \sim( \pm 1 / 3 k \pm 1 / 4)$ is observed near the Bragg spots, where $k$ denotes negligible coherence between the ET layers; that is, the diffuse modulation evolves in two dimensions. This is a clear indication of two-dimensional charge clusters comprised of a $3 \times 4$-period charge order and cannot represent the nucleation of the horizontal charge order with $\mathbf{q}_{\mathrm{o}}\left[=\left(\begin{array}{lll}0 & 0 & 1 / 2\end{array}\right)\right]$, which is almost absent above $200 \mathrm{~K}$; in fact, the $\mathbf{q}_{\mathrm{d}}$ and $\mathbf{q}_{\mathrm{o}}$ modulations compete with each other, as demonstrated in the literature $\mathrm{e}^{20}$. The new feature shown in this study is the temperature profile of the charge clusters. In Fig. 3b,c, the evolution of both the intensity and the correlation length $\xi$ as a function of temperature can be observed, although the $\mathbf{q}_{\mathrm{d}}$ modulation is replaced with $\mathbf{q}_{\mathrm{o}}$ below $200 \mathrm{~K}$ because of the emergence of the twofold horizontal charge order through the first-order transition. The value of $\xi$ is not short-ranged, but it is $\sim 140 \AA$ at $210 \mathrm{~K}$, which corresponds to $\sim 25$ triangular spacings. This behaviour is essentially distinct from conventional critical phenomena, where $\xi$ diverges for a continuous transition. On the basis of a comparison of Figs $3 c$ and 2e, the growth of the slow dynamics and of $\xi$ seem well correlated, indicating that the charge clusters cause the heterogeneous slow dynamics. We also note that of the many different ideas regarding the liquid-glass transition in supercooled normal liquids, our experimental observations for the charge liquid agree phenomenologically with the proposals that consider the crystalline clusters to be the main source of heterogeneous slow dynamics ${ }^{7-10}$.

All of the physical properties discussed so far indicate that the charge-liquid phase above $200 \mathrm{~K}$ is transforming into a chargecluster glass. Thus, the last issue to be studied is the glass transition of the charge clusters, which is expected to occur only if the frustration-relaxing transition at $200 \mathrm{~K}$ is avoided by rapid cooling (Fig. 1c; see also Supplementary Information). A charge-cluster glass transition is observed in the $\xi$ temperature profile measured during heating after rapid cooling $\left(\sim 90 \mathrm{~K} \mathrm{~min}^{-1}\right.$; Fig. 3d). From 120 to $150 \mathrm{~K}, \xi$ is temperature insensitive with a significantly shorter length than expected (see also Fig. 3c), and no superlattice reflections attributable to a deformation of the triangular lattice are observed. This frozen metastable state with no long-range order is characteristic of a glassy state, indicating that a charge-cluster glass is formed in the charge-frustrated triangular lattice. On further heating, $\xi$ increases sharply to the expected value at $T_{\mathrm{g}} \sim 160-170 \mathrm{~K}$ and becomes temperature dependent for temperatures above $T_{\mathrm{g}}$. This behaviour demonstrates that the charge-liquid nature is recovered above $T_{\mathrm{g}}$, and $T_{\mathrm{g}}$ can therefore be regarded as the charge-cluster glass-transition temperature. The temperature evolution of $\xi$ is not critical-like above $T_{\mathrm{g}}$, suggesting that what occurs at $T_{\mathrm{g}}$ is not a thermodynamic transition but of dynamic origin (see also Supplementary Information). Interestingly, previous a.c. conductance measurements reported a broad anomaly at approximately $167 \mathrm{~K}$ during rapid cooling ${ }^{19}$, which we think is another indication of the chargecluster glass transition. The observations of frozen charge clusters, glass-transition behaviour, and glass-forming characteristics of the charge-liquid phase (that is, the heterogeneous slow dynamics) demonstrate that a charge liquid confined to a charge-frustrated triangular lattice is subject to charge-cluster vitrification in an organic system. As the frustration-relaxing structural transition interrupts the charge vitrification process, we suggest that the charge frustration plays a primary role in the charge-glass-forming nature.

Inhomogeneous electronic states are often found in strongly correlated electron systems that contain dopants ${ }^{11,12,30,31}$, such as manganites and high-transition-temperature cuprates, in which randomly distributed dopants are considered to play a key role in the emergent inhomogeneity ${ }^{32,33}$. This issue has been investigated systematically in manganites ${ }^{12,32,34}$, where ferromagnetic metal and
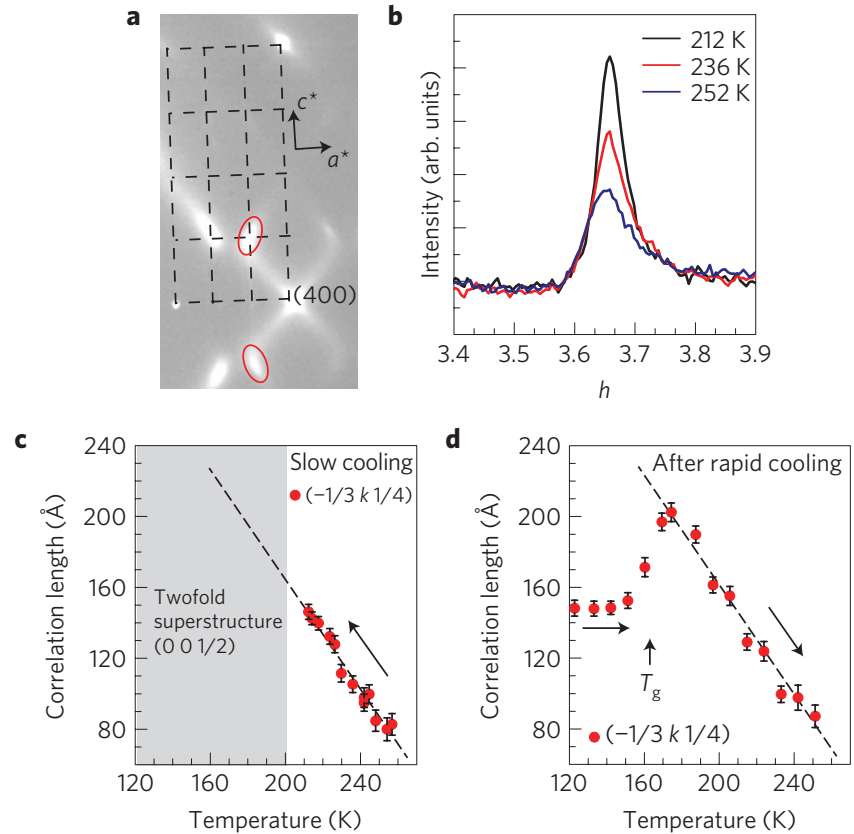

Figure 3 | Spatial correlation of the charge clusters investigated using

X-rays. a, Photographic oscillation image of the $a^{*}-c^{*}$ plane at $225 \mathrm{~K}$. Diffuse rods characterized by $\mathbf{q} \sim( \pm 1 / 3 k \pm 1 / 4)$ are observed near the Bragg reflections (some are indicated by red ellipsoids in the image). $\mathbf{b}$, Line profile of $\mathbf{q} \sim(11 / 3 k 1 / 4)$ along the $-2 a^{*}+c^{*}$ direction. The slight asymmetry in the line shape is due to the background asymmetry, which is temperature independent and irrelevant to the temperature-dependent diffuse scattering. $\mathbf{c}, \mathbf{d}$, Temperature dependence of the charge-cluster correlation length $\xi$ during slow cooling (c) and during heating after rapid cooling to $120 \mathrm{~K}(\mathbf{d})$. The value of $\xi$ is estimated along the $-2 a^{*}+c^{*}$ direction on the $(11 / 3 k 1 / 4)$ diffuse rod. The broken lines in $\mathbf{c}, \mathbf{d}$ are drawn as guides for the eyes. The error bars represent the numerical ambiguity of the fitting.

charge ordered insulator phases are competing and separated by a first-order transition when nominally disorder-free. The two phases, when perturbed by quenched disorders, result in a mixture in a mesoscopic scale, which is the inhomogeneity widely observed in manganites. Conversely, the organic conductor used in this study is nominally dopant-free, and the charge clusters that show up above the first-order transition temperature $(\sim 200 \mathrm{~K})$ are not attributable to a phase mixture, because their symmetry is different from that of the low-temperature charge ordered phase. These situations imply that the microscopic mechanism of the chargecluster glass formation is conceptually distinct from the mechanism discussed in manganites.

\section{Methods}

Crystal growth. Single crystals of $\theta$-(BEDT-TTF $)_{2} \mathrm{RbZn}(\mathrm{SCN})_{4}$ were synthesized by the galvanostatic anodic oxidation of BEDT-TTF $(50 \mathrm{mg})$ in a $\mathrm{N}_{2}$ atmosphere, in accordance with ref. 13 .

Noise measurements. The noise measurements were conducted using the conventional four-terminal d.c. method. A steady current along the $c$ axis was supplied from a low-noise voltage source, and the voltage between the voltage-probing electrodes was fed into a spectrum analyser (Agilent 35670A) after amplifying it with a low-noise preamplifier. A large-load resistor was used to eliminate the resistance fluctuation effect at the contacts. For the material used in this study, this method cannot be applied below $200 \mathrm{~K}$ because the centre frequency $f_{0}$ is too slow; the relaxation from the supercooled charge liquid to the more stable horizontal charge order occurs before the measurements are completed.

Diffraction scattering experiments. The diffraction of a $\theta$-RbZn single crystal $\left(0.4 \times 0.3 \times 2.5 \mathrm{~mm}^{3}\right)$ was measured using a Rigaku DSC imaging plate diffractometer and $\mathrm{Si}(111)$ monochromatized synchrotron radiation $\mathrm{X}$-rays 
$(\lambda=0.689 \AA)$ at the BL-8B beamline of the Photon Factory (PF) at the High Energy Accelerator Research Organization (KEK). The correlation length of the charge clusters is defined as $\xi=\mathrm{FWHM}^{-1}$, where FWHM $\left(\AA^{-1}\right)$ denotes the height of the full-width at half-maximum of the Lorentzian fit to the data.

\section{Received 11 January 2013; accepted 29 April 2013; published online} 9 June 2013

\section{References}

1. Balents, L. Spin liquids in frustrated magnets. Nature 464, 199-208 (2010).

2. Ramirez, A. P., Hayashi, A., Cava, R. J., Siddharthan, R. \& Shastry, B. S. Zero-point entropy in 'spin ice'. Nature 399, 333-335 (1999).

3. Bramwell, S. T. \& Gingras, M. J. P. Spin ice state in frustrated magnetic phyrochlore materials. Science 294, 1495-1501 (2001).

4. Bouchaud, J. P., Cugliandolo, L., Kurchan, J. \& Mezard, M. in Spin-Glasses and Random Fields (ed. Young, A. P.) (World Science, 1998).

5. Anderson, P. W. Ordering and antiferromagnetism in ferrites. Phys. Rev. 102, 1008-1013 (1956)

6. Merino, J., Seo, H. \& Ogata, M. Quantum melting of charge order due to frustration in two-dimensional quarter-filled system. Phys. Rev. B 71, 125111 (2005).

7. Doye, J. P., Wales, D. J., Zetterling, F. H. M. \& Dzugutov, M. The favored cluster structures of model glass formers. J. Chem. Phys. 118, 2792-2799 (2003).

8. Widmer-Cooper, A., Harrowell, P. \& Fynewever, H. How reproducible are dynamic heterogeneities in a supercooled liquid? Phys. Rev. Lett. 93, 135701 (2004).

9. Shintani, H. \& Tanaka, H. Frustration on the way to crystallization in glass. Nature Phys. 2, 200-206 (2006).

10. Tanaka, H., Kawasaki, T., Shintani, H. \& Watanabe, K. Critical-like behaviour of glass-forming liquids. Nature Mater. 9, 324-331 (2010).

11. Dagotto, E. Complexity in strongly correlated electronic systems. Science 309, 257-262 (2005).

12. Tokura, Y. Critical features of colossal magnetoresistive manganites. Rep. Prog. Phys. 69, 797-851 (2006).

13. Mori, H., Tanaka, S. \& Mori, T. Systematic study of the electronic state in $\theta$-type BEDT-TTF organic conductors by changing the electronic correlation. Phys. Rev. B 57, 12023-12029 (1998).

14. Mori, T. Non-stripe charge order in the $\theta$-phase organic conductors. J. Phys. Soc. Jpn 72, 1469-1475 (2003).

15. Seo, H. Charge ordering in organic ET compounds. J. Phys. Soc. Jpn 69, $805-820$ (2000).

16. Watanabe, M., Noda, Y., Nogami, Y. \& Mori, H. Transfer integrals and the spatial pattern of charge ordering in $\theta$-(BEDT-TTF $)_{2} \operatorname{RbZn}(\mathrm{SCN})_{4}$ at $90 \mathrm{~K}$. J. Phys. Soc. Jpn 73, 116-122 (2004).

17. Miyagawa, K., Kawamoto, A. \& Kanoda, K. Charge ordering in a quasi-two-dimensional organic conductor. Phys. Rev. B 62, R7679-R7682 (2000)

18. Yamamoto, K., Yakushi, K., Miyagawa, K., Kanoda, K. \& Kawamoto, A. Charge ordering in $\theta$-(BEDT-TTF $)_{2} \mathrm{RbZn}(\mathrm{SCN})_{4}$ studied by vibrational spectroscopy. Phys. Rev. B 65, 085110 (2002).

19. Nad, F., Monceau, P. \& Yamamoto, H. M. Effect of cooling rate on charge ordering in $\theta$-(BEDT-TTF $)_{2}$ RbZn(SCN $)_{4}$. Phys. Rev. B 76, 205101 (2007).

20. Nogami, Y. et al. Charge order competition leading to nonlinearity in organic thyristor family. J. Phys. Soc. Jpn 79, 044606 (2010).

21. Tajima, H., Kyoden, S., Mori, H. \& Tanaka, S. Estimation of charge-ordering patterns in $\theta-\mathrm{ET}_{2} \mathrm{MM}^{\prime}(\mathrm{SCN})_{4}\left(\mathrm{MM}^{\prime}=\mathrm{RbCo}, \mathrm{RbZn}, \mathrm{CsZn}\right)$ by reflection spectroscopy. Phys. Rev. B 62, 9378-9385 (2000).
22. Chiba, R., Hiraki, K., Takahashi, T., Yamamoto, H. M. \& Nakamura, T. Extremely slow charge fluctuations in the metallic state of the two-dimensional molecular conductor $\theta$-(BEDT-TTF $)_{2} \operatorname{RbZn}(\mathrm{SCN})_{4}$. Phys. Rev. Lett. 93, 216405 (2004).

23. Müller, J. Fluctuation spectroscopy: A new approach for studying low-dimensional molecular metals. ChemPhysChem 12, 1222-1245 (2011).

24. Dutta, P. \& Horn, P. M. Low-frequency fluctuations in solids: $1 / f$ noise. Rev. Mod. Phys. 53, 497-516 (1981).

25. Ediger, M. D. Spatially heterogeneous dynamics in supercooled liquids. Annu. Rev. Phys. Chem. 51, 99-128 (2000).

26. Debenedetti, P. G. \& Stillinger, F. H. Supercooled liquids and the glass transition. Nature 410, 259-267 (2001).

27. Toninelli, C., Wyart, M., Berthier, L., Biroli, G. \& Bouchaud, J-P. Dynamical susceptibility of glass formers: Contrasting the predictions of theoretical scenarios. Phys. Rev. E 71, 041505 (2005).

28. Adam, G. \& Gibbs, J. H. On the temperature dependence of cooperative relaxation properties in glass-forming liquids. J. Chem. Phys. $\mathbf{4 3}$, 139-146 (1965).

29. Kurita, R. \& Weeks, E. R. The glass transition of two-dimensional binary soft disk mixtures with large size ratios. Phys. Rev. E 82, 041402 (2010).

30. Emery, V. J. \& Kivelson, S. A. Frustrated electronic phase separation and high-temperature superconductors. Physica C 209, 597-621 (1993).

31. Schmalian, J. \& Wolynes, P. G. Stripe glasses: Self-generated randomness in a uniformly frustrated system. Phys. Rev. Lett. 85, 836-839 (2000).

32. Akahoshi, Y. et al. Random potential effect near the bicritical region in perovskite manganites as revealed by comparison with the ordered perovskite analogs. Phys. Rev. Lett 90, 177203 (2003).

33. Zeljkovic, I. et al. Imaging the impact of single oxygen atoms on superconducting $\mathrm{Bi}_{2+y} \mathrm{Sr}_{2-y} \mathrm{CaCu}_{2} \mathrm{O}_{8+x}$. Science 337, 320-323 (2012).

34. Dagotto, E., Hotta, T. \& Moreo, A. Colossal magnetoresistant materials: The key role of phase separation. Phys. Rep. 344, 1-153 (2001).

\section{Acknowledgements}

We thank H. Tanaka, K. Kuroki, H. Seo, M. Watanabe, H. Sakai, R. Kurita, Y. Kohsaka, M. Udagawa, T. Itou and M. Imada for fruitful discussions. T.S. was supported by the JSPS Program for Leading Graduate Schools (ALPS). This work has been performed under the approval of the Photon Factory Program Advisory Committee (Proposal No. 2012G115). This work was partially supported by the Funding Program of World-Leading Innovative R\&D on Science and Technology (FIRST program) on Quantum Science on Strong Correlation initiated by the Council for Science and Technology Policy, Japan, and by JSPS KAKENHI (Grant Nos 24684020, 24224009, 20110002, and 24654101)

\section{Author contributions}

F.K. and T.S. conducted the noise measurements. T.S., K. Kobayashi, R.K., Y.M., K.M. and F.K. conducted the X-ray measurements. K.M. grew the single crystals used for the study. F.K. and K. Kanoda planned and headed the project. F.K. wrote the letter with assistance from Y.T. and K. Kanoda.

\section{Additional information}

Supplementary information is available in the online version of the paper. Reprints and permissions information is available online at www.nature.com/reprints. Correspondence and requests for materials should be addressed to F.K.

\section{Competing financial interests}

The authors declare no competing financial interests. 